\title{
Die Habsburg-Lothringische Familien-Fideikommissbibliothek vom Tod Kaiser Franz I. 1835 bis zu ihrer Eingliederung in die Nationalbibliothek der Republik Österreich 1921
}

\author{
Ein Forschungsbericht
}

https://doi.org/10.1515/bfp-2018-0014

Zusammenfassung: Im Rahmen eines Projekts des Österreichischen Fonds zur Förderung der wissenschaftlichen Forschung wird die Geschichte der Familien-Fideikommissbibliothek des Hauses Habsburg-Lothringen aufgearbeitet, deren Bestände sich größtenteils in ihrer historischen Aufstellung noch heute in der Österreichischen Nationalbibliothek befinden. Der vorliegende Artikel gibt über Forschungsschwerpunkte und bisher vorliegende Projektergebnisse Auskunft.

Schlüsselwörter: Bibliotheksgeschichte; Kulturgeschichte; Fideikommiss; Ferdinand I; Franz Joseph I

The Familien-Fideikommissbibliothek of Habsburg-Lorraine from the Death of Emperor Franz I. until it's Incorporation into the National Library of the Republic of Austria 1921 A Project Report

Abstract: A research project financed by the Austrian Science Fund is dedicated to the history of the Familien-Fideikommissbibliothek of Habsburg-Lorraine, which has been mostly conserved so far within is historical locations as part of the Austrian National Library. The following article will give an overview on the objectives and results of this investigation.

Keywords: Library history; cultural studies; fee tail; Ferdinand I; Franz Joseph I

\section{Einleitung}

Das Forschungsvorhaben „Die Habsburg-Lothringische Fideikommissbibliothek 1835-1921. Metamorphosen einer Sammlung“ (P26943, Laufzeit 2014-2018) ist als Folgeprojekt zur Rekonstruktion der „Geschichte der Privatbibliothek Kaiser Franz' I. 1784-1835“ (P22774) zu verstehen und wird wie diese vom Österreichischen Fonds zur Förderung der wissenschaftlichen Forschung finanziert. ${ }^{1}$ Das Hauptaugenmerk liegt zum einen auf der Transformierung dieser einzigartigen Sammlung zu einer Fideikommissbibliothek und dem Zusammenschluss mit anderen kaiserlichen Privatbibliotheken (Ferdinands I. und Franz Josephs I.) zur Habsburg-Lothringischen Familienbibliothek. Darüber hinaus wird im Zuge der Projektarbeit aber auch versucht, jene Suche nach der eigenen Identität und nach dem Zweck der Sammlung herauszuarbeiten, die während des Fin de Siècle vor dem Hintergrund des zunehmenden öffentlichen Interesses und der für den Weiterbestand der Monarchie unerlässlichen Loyalität gegenüber dem Kaiserhaus die Leiter und Beamten der Fideikommissbibliothek beschäftigte. $\mathrm{Zu}$ guter Letzt wird die Situation während des Ersten Weltkriegs, seine Auswirkung auf das Personal und die in dieser Phase gemachten Bestandszuwächse beleuchtet.

\section{Die Privatbibliothek Ferdinands I.}

Eine parallel zur Privatbibliothek Franz' I. entstandene Buch- und Kunstsammlung ist die seines ältesten Sohnes und Regierungsnachfolgers Ferdinand (1793-1875). In Ermangelung adäquater Quellen kann auf Grundlage des

1 Die Forschungsergebnisse des ersten Projekts sind publiziert in Huber-Frischeis, Knieling und Valenta (2015) und Huber-Frischeis, Knieling und Valenta (2014).
*Kontaktpersonen: Nina Knieling, knieling@ponte.at Thomas Huber-Frischeis, thomas.huber@onb.ac.at RainerValenta, rainer.valenta@onb.ac.at Hans Petschar, Hans.Petschar@onb.ac.at 
vorhandenen Archivmaterials über die Genese und Funktion dieser im Vergleich zur väterlichen Sammlung überschaubaren Bibliothek nur spekuliert werden. Sie hatte vermutlich nicht nur eine ähnliche Entstehungsgeschichte, sondern auch dieselben Aufgaben. ${ }^{2}$ Mit dem Regierungsantritt Ferdinands übernahm seine Büchersammlung die Rolle der franziszeischen Privatbibliothek, da sie von nun an alle Werke, die dem Kaiser von Autoren, Künstlern, Verlegern etc. überreicht wurden, zur Aufbewahrung zugeteilt erhielt. Nebenher wuchs die Sammlung durch gezielte Ankäufe von Werken, die das Interesse des für nur bedingt regierungsfähig gehaltenen Kaisers hervorriefen. So geringschätzig Ferdinand in der österreichischen Geschichtsschreibung seit jeher behandelt wird, so stiefmütterlich ging man damals schon mit seiner Bibliothek um. Seit jeher auf mehrere Standorte in und um seine Wohnappartements verteilt, vagabundierten die Bücherkästen vor und während seiner Regierungszeit aufgrund ständiger Raumumwidmungen anlässlich Einquartierung von Gästen. Eine physische Zusammenführung mit der Fideikommissbibliothek und Aufstellung in deren Räumlichkeiten schien aus Platzgründen unmöglich zu sein. Die Ereignisse 1848 führten im Zuge der Thronentsagung schließlich zum dauerhaften Umzug Ferdinands nach Prag, wohin er seine Bibliothek mitnahm. Sie wurde dort untergebracht im Toskana-Palais gegenüber der Prager Burg - vom Geistlichen Nikolaus Negrelli und einem Bibliotheksdiener verwaltet. ${ }^{3}$ Testamentarisch vermachte Ferdinand seine Sammlung, die 1875 nach Wien zurückkehrte, seinem Neffen Franz Joseph.

\section{Die Privatbibliothek Franz Josephs I.}

Anders als seine Vorgänger, war Franz Joseph kein Bibliophile. Während über den Nukleus dieser Sammlung wiederum nur Vermutungen ausgesprochen werden können, nimmt sie, was den Buchbestand betrifft, erst substanzielle Gestalt an, als Franz Joseph 1848 Kaiser von Österreich wird. Dies allerdings nur theoretisch, weil Franz Joseph die an ihn gerichteten Buchgeschenke bis 1870 zunächst durch

2 Etwa zu den Rechnungen Kaiser Franz' I. über seine eigenen Ausgaben und jene für seine Kinder, Wien, Österr. Staatsarchiv, Haus-, Hof- und Staatsarchiv, Gen. dir. d. ah. Privat- u. Fam.fonde Ä.R., Kts. 44-58 (Rechnungen der Kinder Franz ' I. von 1792-1810) und Kts. 72102 (1792-1820) Privatrechnungen und Kammerausgaben Franz ' I.).

3 Vgl dafür den Bestand Prag, Narodni Archiv, Urad nejvyssiho dvorskeho hofmistra cisare Ferdinanda I. die Hofbibliothek verwalten lässt. Erst Bibliotheksvorstand Moritz Alois von Becker gelingt es diese Entscheidung rückwirkend zu korrigieren und die franzisko-josephinische Privatbibliothek erhielt schließlich jene (seit 1849) eingelangten Bestände zurück, die ihr aufgrund ihrer Funktion zustanden. Seine Privatbibliothek wurde zunächst im Dachgeschoss oberhalb der Fideikommissbibliothek untergebracht und bestand (vor der Retournierung der Bücher aus der Hofbibliothek) großteils aus individuell angefertigten, großformatigen Huldigungsadressen, für deren Aufbewahrung sich die dortigen Bücherregale als ungeeignet erwiesen. Das große Raumvolumen dieser behelfsmäßigen Herberge verhinderte darüber hinaus eine Beheizung in den Wintermonaten, weshalb etwa Katalogisierungs- oder Bestandserhaltungsmaßnahmen nur während der wärmeren Jahreshälfte stattfinden konnten. Mit dem Tod Ferdinands I. 1875 und dem Übergang seiner Privatbibliothek in den Besitz Franz Josephs erhält dessen Büchersammlung ihren größten und bedeutendsten $\mathrm{Zu}$ wachs. Unter Beckers umsichtiger Führung, wird schlussendlich die Aufstellung zumindest der ferdinandeischen Bestände in den Räumen der Fideikommissbibliothek möglich.

\section{Die Fideikommissbibliothek und ihr wechselvolles Schicksal}

Mit dem Tod Franz' I. am 2. März 1835 ließ Kaiser Ferdinand Neuerwerbungen für die franziszeische Privatbibliothek einstellen. Lediglich die große Anzahl an Fortsetzungswerken sowie Neuerscheinungen, die den Bestand maßgeblich ergänzten, durften auf Kosten der Bibliotheksdotation weiterhin bezogen respektive ergänzt werden. Die Buch- und Kunstsammlung des verstorbenen Kaisers wurde gewissermaßen als abgeschlossene Einheit betrachtet, die sich so zu einem bibliophilen Denkmal für ihren Gründer transformierte. Die frei gewordenen finanziellen wie personellen Ressourcen kamen nun der Aufarbeitung der Ferdinandea zugute. Eine Zusammenführung beider Bestände wurde zwar mittelfristig ins Auge gefasst, war aber aufgrund unterschiedlicher Katalogisierungstiefen und angesichts des von Bibliotheksvorsteher Leopold Joseph von Khloyber stets als bedrückend geschilderten Raummangels vorerst nicht möglich.

Die Ferdinand übertragene Aufgabe, den zwölften Paragrafen im Testament Franz ' I. zu vollstrecken und dessen Sammlungen durch die Überführung in einen Primogenitur-Fideikommiss dauerhaft für die Nachkommenschaft als Entität zu bewahren, konnte während seiner Regie- 
rungszeit nicht zustande gebracht werden. Der Grund hierfür lag etwa bei Auffassungsunterschieden in der Frage, ob nur die im Testament explizit erwähnten Sammlungsbestandteile dem Fideikommiss zuzurechnen wären - der Wille des Kaiser also wortwörtlich auszulegen sei - oder ob man all jene Gegenstände, die de facto Teil der franziszeischen Privatbibliothek waren - also auch Gemälde, Münzen, Notendrucke etc. - miteinbeziehen sollte.

Erst Franz Joseph ist das Verdienst zuzuschreiben, die überkommenen bürokratischen Altlasten einer Erledigung zuzuführen. Die Fideikommiss-Errichtungsurkunde wurde bereits im August 1849 aufgesetzt, obwohl die Inventare, in denen jedes einzelne inkorporierte Objekt verzeichnet werden sollte, noch nicht fertiggestellt war. Zur Klärung strittiger Punkte hatte man eine eigene Kommission eingesetzt. Erst eine Dekade später konnte anhand der schließlich vollendeten Verzeichnisse eine gerichtliche Inventur vorgenommen werden. Zeitgleich wurden das Obersthofmarschallamt als Fideikommissbehörde und Erzherzog Ludwig (1784-1864, ein jüngerer Bruder Kaiser Franz' I.) als erster Fideikommisskurator eingesetzt. ${ }^{4}$

Den Mangel an bibliophilen Interessen kompensierte der junge Kaiser nicht nur mit tatkräftigen Maßnahmen, sondern auch mit richtungsweisenden Personalentscheidungen, wie die, den Lehrer Kronprinz Rudolfs, Moritz Alois von Becker, zum Nachfolger des 186. verstorbenen Bibliotheksvorstehers Khloyber zu ernennen.

Hatte die Fideikommissbibliothek die Ferdinandea 1850 nach Prag verloren, so erwuchs ihr mit der sukzessiv anwachsenden Privatbibliothek Franz Josephs eine neue. Beckers anlässlich des Dienstantritts 1870 verfasster Bericht samt Arbeitsprogramm ist ein eindrucksvolles Zeugnis des damaligen Zustandes dieser Sammlungen und enthält seine richtungsweisenden Visionen, wie sich die Bestände entwickeln und was ihr zukünftiger Zweck sein sollten. ${ }^{5}$

Die Entscheidungen fällte Franz Joseph in seiner Funktion als Oberhaupt der kaiserlichen Familie und als Herrscher. Fideikommissinhaber wurde er den Nachfolgeregelungen des Hauses Habsburg-Lothringen allerdings erst, als sein Vater Erzherzog Franz Karl 1878 verstarb. Franz Karl war seit 1875 Fideikommissherr, vor ihm Kaiser Ferdinand I. seit $1849 .{ }^{6}$ Sowohl Onkel als auch Vater hat-

4 Auf ihn folgte Erzherzog Leopold (1823-1898, ältester Sohn Erzherzog Rainers) und schließlich Erzherzog Eugen (1863-1954, ein Enkel des Aspern-Siegers Erzherzog Karl).

5 Wien, Österreichische Nationalbibliothek, Bildarchiv und Grafiksammlung, Archiv der Fideikommissbibliothek, FKBA26135.

6 Ferdinand war freilich schon seit dem Tod seines Vaters Franz 1835 Besitzer dessen Privatbibliothek, doch erst 1849 wurde er durch die ten Franz Joseph allerdings weitreichende Entscheidungsund Handlungsfreiheit in diesen Belangen zuerkannt.

Gravierende Folgen hatte der Umstand, dass auf Beckers Vorschlag hin mit der Erstellung eines gedruckten Realkataloges begonnen wurde - eine Initiative zur erstmaligen Präsentation und Bekanntmachung der Sammlung sowohl unter den weitverzweigten Mitgliedern des Kaisershauses, als auch in anderen fürstlichen und öffentlichen Bibliotheken. ${ }^{7}$ Letztere waren im Hinblick auf die Bestandserweiterung vorrangig als Tauschpartner zur Verwertung von Dubletten von großem Interesse. Das Katalogprojekt blieb jedoch unvollendet, da der letzte Teil zur Porträt- und Kunstsammlung nie wirklich in Angriff genommen wurde.

\section{Eine große Familien-Sammlung entsteht}

Nach dem Tod seines Vaters Erzherzog Franz Karl im Jahre 1878 wurde Kaiser Franz Joseph Fideikommissherr. Der Monarch war nun, im dreißigsten Jahr seiner Regentschaft, endlich offizieller und alleiniger Besitzer der Fideikommissbibliothek geworden, deren Entwicklung er bereits zuvor maßgeblich beeinflusst hatte. In den nachfolgenden dreieinhalb Jahrzehnten bis zum Ersten Weltkrieg erlebte die Sammlung eine Reihe von entscheidenden Veränderungen und Neuerungen in unterschiedlichen Bereichen.

Der erste wichtige Schritt war die Vereinigung der Privatbibliothek des Kaisers mit der Fideikommissbibliothek, die infolgedessen ab 1878 den Namen „k. k. [seit 1889: „k. u. k.] Familien-Fideikommissbibliothek" führte. Administrativ bedeutete dies neben der Zusammenlegung der Bestände auch gemeinsame Katalogisierung und Finanzierung sowie Doublettenabgleich und -verkauf. Zugleich wurden die jährlich in großer Zahl abgegebenen persönlichen Widmungen an den Kaiser zu einem bestimmenden Faktor im Bestandszuwachs. Gezielte Ankäufe wurden hauptsächlich zwecks Ergänzung und Vervollständigung der Bestände getätigt, wobei seit der Jahrhundertwende der Fokus fast nur mehr auf dem Erwerb von Austriaca lag.

Von entscheidender Bedeutung für die Administration der Fideikommissbibliothek war die Schaffung einer Generaldirektion für alle dem habsburgischen Familienfonds zugehörigen Güter und Einrichtungen im Jahr 1886. Dieser

Ausstellung der entsprechenden Urkunde auch formell Fideikommissherr.

7 Becker (1873), Becker (1875), Becker (1879) und Becker (1882). 
Behörde war die Sammlungsleitung ab da direkt untergeordnet und in allen der Genehmigung bedürftigen Angelegenheiten Rechenschaft schuldig. Ab 1890 fungierte der zuvor in der Finanzverwaltung tätige Emil Freiherr von Chertek als Generaldirektor des k. u. k. Familienfonds. Aus der Aktenkorrespondenz geht hervor, dass Chertek ein sehr kontrollierender Charakter mit ausgesprochenem Sinn für Kostenreduktion war, und das hatte notgedrungener Weise auch Auswirkungen auf die Verwaltung der Fideikommissbibliothek.

Nach dem Tod des pensionierten Universitätsprofessors Joseph von Zhishman 1894, der 1887 die Nachfolge Beckers als Direktor der Fideikommissbibliothek angetreten hatte, ließ Chertek den Posten nicht mehr nachbesetzen. ${ }^{8}$ Stattdessen wurde die Leitung der Bibliothek dem ersten Kustos Alois Karpf übertragen. Diesem war es jedoch aufgrund seines schlechten Verhältnisses zum Generaldirektor und seiner schwachen Stellung unter den Mitarbeitern kaum möglich Akzente in der Sammlung zu setzen. Nach mehreren für Karpf erniedrigenden Vorkommnissen zu Beginn des 20. Jahrhunderts suchte er im Sommer 1906 schließlich um seine Pensionierung an. Sein Nachfolger wurde Franz Schnürer, der bereits seit längerem in einem engen Vertrauensverhältnis zu Chertek gestanden und mit verschiedenen Initiativen die Geschicke der Sammlung beeinflusst hatte (s.u.).

\section{6 Über mehrere provisorische Standorte zur heutigen Aufstellung}

Im vorliegenden Zeitabschnitt erlebte die Fideikommissbibliothek insgesamt drei Übersiedlungen. Becker hatte die Lösung des Raumproblems, um die sich Khloyber jahrzehntelang vergeblich bemüht hatte, gleich bei seinem Amtsantritt als eine der dringendsten Angelegenheiten erkannt. Die vorgesehenen Räumlichkeiten im Augustinergang (einem Gebäudetrakt neben der Hofbibliothek) konnten jedoch erst 1890/91 bezogen werden, nachdem die zuvor dort befindlichen Hofsammlungen in die neuen $\mathrm{Mu}$ seumsgebäude am Ring übersiedelt worden waren. Der neue Standort war aber nur provisorisch, da der Augustinergang im Zuge des Hofburgneubaues abgerissen werden

\footnotetext{
8 Zhishman war Professor für Kirchenrecht und wie Becker ein ehemaliger Lehrer der Kronprinzen Rudolf. Nach seinem Tod hatten sich acht Personen für seine Nachfolge beworben, von denen die meisten angaben, Lehrer von Erzherzogen oder Erzherzoginnen gewesen zu sein. Zu Zhishman siehe Hamann (1991) 55-59.
}

sollte. Nach Erwägung mehrerer Alternativvorschläge (Unteres Belvedere, Augustinertrakt der Hofburg) wurde die Fideikommissbibliothek 1903 in Räumen im Hochparterre und im Souterrain des Corps de logis der eben fertiggestellten Neuen Burg aufgestellt. Doch auch diese Unterbringung erwies sich nicht als dauerhaft, da das Bücherdepot im Souterrain durch Feuchtigkeitsschäden und Schimmelpilzbefall bedroht war. Nach mehreren Interventionen Schnürers erhielt die Fideikommissbibliothek im Jahr 1908 schließlich ihre definitive, bis heute bestehende Unterbringung im zweiten Obergeschoss des Corps de logis. ${ }^{9}$

\section{Die Fideikommissbibliothek gewinnt die Aufmerksamkeit der Öffentlichkeit}

In der Periode zwischen 1878 und 1914 entwickelte sich die Fideikommissbibliothek von einer wenig bekannten, unzugänglichen Privatsammlung der kaiserlichen Familie zu einer in vielfältiger Weise in die Öffentlichkeit wirkenden und von dieser genutzten Institution. Die wesentlichste Voraussetzung dafür war der von Moritz Alois Becker initiierte gedruckte Katalog, der die Bestände der Sammlung allgemein bekannt machte und das Interesse für diese weckte.

Die Möglichkeiten der öffentlichen Nutzung der Bestände waren vielfältig, eine davon ergab sich im Ausstellungswesen. Bis zum Ausbruch des Ersten Weltkrieges hat die Fideikommissbibliothek Leihgaben für mehr als siebzig Ausstellungen zur Verfügung gestellt, teilweise auch außerhalb der Monarchie. Zu Beginn spielte dabei vor allem eine Objektklasse eine bevorzugte Rolle: die sogenannten Huldigungsadressen. Als Loyalitätsbekundungen an den Kaiser und seine Familie und vor allem als hervorragende Schöpfungen der österreichischen Kunstindustrie wurden sie zahlreiche Male in der Öffentlichkeit präsentiert. ${ }^{10}$ Dies erfolgte v.a. im neugegründeten Museum für Kunst und Industrie, dessen Direktor Rudolf von Eitelberger eine bedeutende Rolle in der Wiener Museumslandschaft spielte. Nach der ersten Übersiedlung wurde in

9 Die Übersiedlungen der Fideikommissbibliothek sind natürlich nur vor dem Hintergrund der komplexen Um- und Neubauten der Hofburg in der zweiten Hälfte des 19. Jahrhunderts zu verstehen; siehe dazu Telesko und Rosenauer (2012) 156-395.

10 Bei den Huldigungsadressen handelt es sich eigentlich um kalligrafisch gestaltete Widmungsschriften. Diese waren jedoch in vielen Fällen in kunstvoll gestaltete Einbände oder Kassetten (sog. „Enveloppes“) eingelegt und somit auch Werke der Kunstindustrie. 
den neuen Räumlichkeiten im Augustinergang zudem für kurze Zeit (1893/94) eine hauseigene Ausstellung präsentiert, innerhalb derer die Huldigungsadressen wiederum die Hauptattraktion darstellten. ${ }^{11}$

Auch zu Forschungszwecken wurde die Sammlung in Anspruch genommen, wobei hier der Fokus in erster Linie bei den Handschriften und bei der Porträtsammlung lag. Diese letztere, die bereits im späten 19. Jahrhundert als eine der größten und bedeutendsten der Welt galt, wurde von Außenstehenden in rasch steigendem Maße auf sehr unterschiedliche Weisen genutzt. Bildnisse dienten neben anderen grafischen Blättern sehr häufig als Vorlagen für Reproduktionen in Büchern. Becker hatte eingeführt, dies für wissenschaftliche, nichtkommerzielle Unternehmungen zu genehmigen, falls das entsprechende Bildmaterial in öffentlichen Sammlungen nicht erhältlich war. Im Laufe der Zeit wurden diese Bestimmungen jedoch zunehmend liberaler gehandhabt.

Blätter aus der Porträtsammlung dienten zudem als Vorlagen für Skulpturen an öffentlichen Bauwerken und Denkmälern sowie für Bildnisgalerien. Ein besonders häufig $\mathrm{zu}$ beobachtendes Phänomen in diesem Zusammenhang sind die Anfragen von Regimentern der k. u. k. Armee, welche die Bildnisse ihrer Inhaber nach Vorbildern aus der Porträtsammlung kopieren ließen.

Die Kehrseite dieser Entwicklung war, dass man in der Fideikommissbibliothek seit ca. 1880 besondere Kompetenzen im Hinblick auf die Bestimmung und bibliografische Beschreibung von Porträts entwickelte. Der Umstand, dass der geplante vierte Band des gedruckten Kataloges aufgrund der unzureichenden Erschließung der Porträtsammlung niemals zustande gekommen war, führte $z u$ einer intensiven Auseinandersetzung mit den eigenen Beständen und zur Anlage von Zettelkatalogen, die dem Bestimmen und Auffinden von Bildnissen dienten. In der gebildeten Öffentlichkeit galt die Fideikommissbibliothek bald als Kompetenzzentrum der Porträtforschung, was sich u.a. in unzähligen Porträtbestimmungen für $\mathrm{Au}$ ßenstehende manifestierte.

11 Die „Musealisierung“ öffentlicher Bibliotheken war in der zweiten Hälfte des 19. Jahrhunderts keine Seltenheit; vgl. Kaltwasser (1999) 214-66.

\section{Der gescheiterte Plan eines „Erinnerungsraumes" für das Haus Habsburg-Lothringen}

Seit ihrer Gründung, jedoch in zunehmendem Maße während der Inhaberschaft Franz Josephs war die Fideikommissbibliothek eine Vereinigungsstätte für Nachlassbestände und persönliche Dokumente von Mitgliedern der Dynastie. Auf das Sammeln von Austriaca und Habsburgica legte zudem Becker sein besonderes Augenmerk. Kurz vor 1900 verfasste Schnürer schließlich ein Regulativ für den Ankauf, das den Schwerpunkt ausschließlich auf Werke mit Bezug zur Monarchie und zum Herrscherhaus legte. ${ }^{12}$ Es wurde von Chertek, wohl auch aus Kostengründen, genehmigt, womit der Fideikommissbibliothek nun dezidiert der Charakter einer Habsburg-lothringischen Familiensammlung verliehen werden sollte. Gleichzeitig entwickelte Schnürer im Rahmen der Vorbereitungen zur zweiten Übersiedlung die Idee, in den neuen Sammlungsräumen ein Habsburgermuseum $\mathrm{zu}$ etablieren. Dieser Plan, der vom Hohenzollernmuseum in Berlin ${ }^{13}$ inspiriert war und nach den Vorstellungen seines Autors dezidiert propagandistische Funktionen erfüllen sollte, stieß bei Chertek und anderen Hofämtern zunächst auf großes Interesse. ${ }^{14}$ Aus unerfindlichen Gründen wurde er bei der Übersiedlung 1903 aber nicht verwirklicht und später auch nicht mehr aufgegriffen. Dabei hätte ein solches Museum wohl Interesse in der Öffentlichkeit wecken können: In der Zeit nach der Jahrhundertwende wurden persönliche Dokumente des Herrscherhauses und v.a. des Kaisers selbst (beispielsweise seine Schulhefte) in zunehmenden Maße für Ausstellungen und für Veröffentlichungen begehrt.

\section{Die Fideikommissbibliothek im Ersten Weltkrieg}

Während des Ersten Weltkriegs kam es zu einschneidenden Veränderungen, die im Besonderen durch die Einberufung des Bibliothekspersonals zum Kriegsdienst unmittelbare Auswirkungen hatten, und sich im Allgemeinen in

12 Wien, Österreichische Nationalbibliothek, Bildarchiv und Grafiksammlung, Archiv der Fideikommissbibliothek, FKBA37193.

13 Siehe zu diesem Biskup und Kohlrausch (2008).

14 Schnürer hat mehrere Denkschriften verfasst, die heute im Haus-, Hof- und Staatsarchiv in Wien liegen: GDPFF, Sonderreihe, Kt. 17, Z. 1450 ex 1899, Z. 3294 ex 1899, Z. 1986 ex 1901; R. 5, Kt. 536, Z. 967 ex 1907. 
das Bild vom Stillstand der wissenschaftlichen Forschung und literarischen Arbeit der Kriegszeit einfügten. ${ }^{15}$ Dies spiegelte sich im Personalstand einerseits und andererseits in den stark reduzierten Anfragen, Entlehnungen und Ausstellungen wider. Gleichzeitig wurde das Bibliothekspersonal ab dem Jahr 1917 durch zwei „weibliche Hilfskräfte“ verstärkt, die rund ein Viertel des Gehalts ihrer männlichen Kollegen auf äquivalenten Posten verdienten, welche sich aber im Dienst an der Front befanden. Trotz der Kriegszeiten kann man die Situation des am Wiener Hof verbliebenen Bibliothekspersonals als sehr abgesichert bezeichnen. Einerseits wurde der zu diesem Zeitpunkt als Kustos an der Bibliothek befindliche Rudolf Payer-Thurn mit der Begründung der Unabkömmlichkeit in der Bibliothek vom Kriegsdienst enthoben. Andererseits wurde die Enthebung und Rückführung in den Bibliotheksdienst für den 1914 als untauglich eingestuften Ernst Hefel schließlich 1917 umgesetzt. Wie schon vor dem Krieg geschehen, wurde das Bibliothekspersonal mit regelmäßigen Aushilfszahlungen versorgt. Dies umfasste die aufgrund der allgemeinen Geldentwertung ausbezahlten Teuerungszulagen und Annualzulagen, aber auch $\mathrm{Zu}$ schüsse von der Generaldirektion der allerhöchsten Privatund Familienfonds, welche zur finanziellen Unterstützung des Personals beispielsweise für deren Kinder ausbezahlt wurden. Als Gründe dafür wurden die Geburt und Ausbildung der Kinder angegeben, aber auch finanzielle Aushilfen für den Urlaub bzw. die Equipierung für den Kriegsdienst wurden getätigt. Die Beamten hatten darüber hinaus Gelegenheit, von den durch die Generaldirektion verwalteten habsburgischen Grundherrschaften Lebensmittel zu beziehen.

Durch die weiblichen Hilfskräfte konnten die Katalogisierungsarbeiten in der Fideikommissbibliothek, die durch die 1908 erfolgte Übersiedlung in den zweiten Stock des Corps de logis der Neuen Hofburg notwendig geworden waren, weitestgehend abgeschlossen werden. Der Bestandsaufbau durch Ankauf kann in den Kriegsjahren als praktisch abgeschlossen bezeichnet werden, da kaum noch Erwerbungen in die Bibliothek gelangten. Dennoch wurde auf Bestreben von Bibliotheksdirektor Franz Schnürer eine eigene Kriegssammlung mit dem Titel „Kaiser und König Karl im Weltkrieg“ angelegt, die in Text- und Bilddokumenten den Erfolg der Österreichisch-Ungarischen Armee festhalten sollte. In diese Sammlung fanden auch dem Kaiser von Einzelpersonen gewidmete Werke Eingang. Darüber hinaus war Franz Schnürer weiterhin der

$15 \mathrm{Zu}$ den Jahren 1914-1921 grundlegend: ÖNB, BAG, Archiv der Fideikommissbibliothek, 42-47.
Motor für eine supranationale und dynastische Musealisierung der Fideikommissbibliothek. Die Pläne für eine permanente Ausstellung wurden von der Generaldirektion allerdings aufgrund des Krieges auf die lange Bank geschoben.

Die Fideikommissbibliothek des Hauses HabsburgLothringen war bis zum Untergang der Monarchie zu keinem Zeitpunkt eine öffentliche Bibliothek. Dennoch fanden Entlehnungen statt, die sich auf Grundlage des Entlehnprotokolls ${ }^{16}$ in drei Gruppen einteilen lassen: Einerseits sorgten die Beamten der Fideikommissbibliothek für die größte Anzahl an Entlehnungen, andererseits scheinen Beamte des Hofstaats oder nahestehender staatlicher Institutionen auf. Des Weiteren gab es regelmäßige Entlehnungen von befreundeten Personen des Bibliothekspersonals, die ebenfalls zum Großteil dem katholisch-konservativen Lager zugeordnet werden können, wie der Schriftsteller Richard Kralik von Mayrswalden oder der Rektor der Universität Wien und Professor für Pastoraltheologie Heinrich Swoboda. Die kaiserliche Familie entlehnte nur vereinzelt Werke, wie dies bei Erzherzogin Marie Valerie oder Kaiserin Zita der Fall war. Grundsätzlich zeigten weder Kaiser Franz Joseph noch der ab 1916 de facto Fideikommissinhaber Kaiser Karl Interesse an der Bibliothek. Die de jure erfolgte Einantwortung der Fideikommissbibliothek fand schließlich auch erst im Kriegsjahr 1918 statt. Dennoch ließ Kaiser Karl noch in demselben Jahr auf Vorschlag von Franz Schnürer eine Büste von Franz Joseph in Auftrag geben, die allerdings erst nach dem Untergang der Monarchie geliefert werden konnte.

\section{Die Fideikommissbibliothek wird zur „Porträtsammlung“}

Mit dem Ende der Republik brach das Fundament der Fideikommissbibliothek über Nacht zusammen. Durch das am 3. April 1919 erlassene und am 30. Oktober desselben Jahres novellierte sogenannte „Habsburgergesetz“ wurde die Familie Habsburg-Lothringen ihres gebundenen Vermögens enteignet. ${ }^{17}$ Diesem durch Fideikommiss gebundenen Vermögen wurde auch die Bibliothek zugeordnet, welche in der zweiten Hälfte des 18. Jahrhunderts von Franz II./I. gegründet und aufgebaut wurde. Derselbe hatte es erst durch den $\S 289$ des 181. in Kraft getretenen „Allgemei-

16 ÖNB, BAG, FKB.INV.64, Entlehnprotokoll der Fideikommissbibliothek.

17 Habsburgergesetz $\S 6$ (2). 
nen Bürgerlichen Gesetzbuches“ der Habsburgermonarchie möglich gemacht „das Vermögen des Landesfürsten, welches er nicht als Oberhaupt des Staates besitzt, [...] als ein Privat-Gut ${ }^{\text {"18 }}$ zu betrachten. Durch den Vertrag von Saint-Germain-en-Laye vom 9. September 1919 wurde auch die Frage nach der Aufteilung von Kulturgütern der Habsburgermonarchie zwischen der Republik Österreich und den Nachfolgestaaten geregelt. Nach der Visitation der Bibliothek durch die Repräsentanten der Nachfolgestaaten gelangten keine Forderungen an den Beständen der Fideikommissbibliothek an die Bibliotheksdirektion, bis auf die Bibliothek Kaiser Ferdinands aus Prag, auf die die Tschechoslowakei Ansprüche stellte. Nach der Stellungnahme des neuen Bibliotheksdirektors Rudolf PayerThurn wurde diese in weiterer Folge abgewiesen. Dieser war auch gegen die Unterstellung der Sammlung unter das Unterrichtsministeriums, weil er die Auflösung der Sammlung befürchtete. Schließlich wurde in der 193. Sitzung des Kabinettsrats vom 18. Juni 1920 die Übernahme der Fideikommissbibliothek in die staatliche Verwaltung der Republik Österreich beschlossen und dem Bundesministerium für Inneres und Unterricht unterstellt. In einem zweiten Schritt erfolgte die Unterstellung in die Verwaltungsstruktur der neugegründeten Nationalbibliothek und die nunmehrige Zugänglichkeit $\mathrm{zu}$ den Beständen für die Allgemeinheit. Die neue republikanische Ära verlangte dabei eine Neuorientierung der Sammlungen und einen sichtbaren Bruch mit der monarchischen Vergangenheit. So fand in der neuen Bestandsbezeichnung weder die Entstehungsgeschichte der Fideikommissbibliothek noch das Haus Habsburg-Lothringen Erwähnung. Ab diesem Zeitpunkt wurde die gesamte Sammlung als „Porträtsammlung“ bezeichnet, obwohl sie nach wie vor den Großteil der übrigen Bestände, wie vor allem die umfassende Büchersammlung beinhaltete. Doch es gab auch einige Abgänge. Unter großem Protest von Payer-Thurn und Schnürer wurde nun von der Generaldirektion der Nationalbibliothek die sogenannte „Zusammenlegung gleicher Sammlungsbestände“ vorangetrieben. Die Handschriften und Inkunabeln der ehemaligen Fideikommissbibliothek gingen an die Handschriftenabteilung der Nationalbibliothek, Landkarten, Pläne, aber auch nach geografischen Gesichtspunkten geordnete Vues gingen an die Kartensammlung. Noten und Musikalien kamen an die Musiksammlung. Die Albertina erhielt Handzeichnungen und Kunstblätter. Im Austausch dafür gelangten die Porträtgrafiken anderer Abteilungen und Institutionen in die Verwaltung der Porträtsammlung. Bei diesem Tauschbasar der Sammlungen wurden Sammlungsbestände zerrissen und es bedarf weiterer Forschungsarbeit, um die Provenienzgeschichte in adäquater Form aufzuarbeiten. Durch die im Rahmen des FWF-Projekts erfolgte Aufarbeitung der Bibliotheksgeschichte ist hier ein erster Schritt gesetzt worden. Die Grundlagenforschung für die Bestandsaufteilungen innerhalb der Abteilungen der Nationalbibliothek stellt allerdings nach wie vor ein Forschungsdesiderat dar.

\section{Literaturverzeichnis}

Allgemeines Bürgerliches Gesetzbuch (1811): Allgemeines Bürgerliches Gesetzbuch: § 289. Verfügbar unter http://alex.onb.ac.a t/cgi-content/alex?aid=jgs\&datum=1012\&page $=505 \&$ size $=45$, zugegriffen am 25.06.2017.

Becker, Moritz Alois (1873): Die Sammlungen der vereinten Familienund Privat-Bibliothek Sr. M. des Kaisers. Wien: Finsterbeck.

Becker, Moritz Alois (1875): Die Sammlungen der vereinten Familienund Privat-Bibliothek Sr. M. des Kaisers. Wien: Finsterbeck.

Becker, Moritz Alois (1879): Die Sammlungen der vereinten Familienund Privat-Bibliothek Sr. M. des Kaisers. Wien: Holzhausen.

Becker, Moritz Alois (1882): Die Sammlungen der vereinten Familienund Privat-Bibliothek Sr. M. des Kaisers. Wien: Holzhausen.

Biskup, Thomas; Kohlrausch, Martin (Hrsg.) (2008): Das Erbe der Monarchie: Nachwirkungen einer deutschen Institution seit 1918. Frankfurt/Main: Campus Verlag.

Hamann, Brigitte (1991): Rudolf: Kronprinz und Rebell. 7. Aufl. Wien, München: Amalthea.

Huber-Frischeis, Thomas; Knieling, Nina; Valenta, Rainer (2014): Die Geschichte der Privatbibliothek Kaiser Franz' I. von Österreich 1784-1835. In: BIBLIOTHEK - Forschung und Praxis, 38 (3), 461-67.

Huber-Frischeis, Thomas; Knieling, Nina; Valenta, Rainer (2015): Die Privatbibliothek Kaiser Franz I. von Österreich 1784 - 1835: Bibliotheks- und Kulturgeschichte einer fürstlichen Sammlung zwischen Aufklärung und Vormärz. Köln: Böhlau Verlag (Veröffe ntlichungen der Kommission für Neuere Geschichte Österreichs: 111).

Kaltwasser, Franz Georg (1999): Die Bibliothek als Museum: Von der Renaissance bis heute, dargestellt am Beispiel der Bayerischen Staatsbibliothek. Wiesbaden: Harrassowitz (Beiträge zum Buchund Bibliothekswesen: 38).

Telesko, Werner; Rosenauer, Artur (2012): Die Wiener Hofburg 1835 1918: Der Ausbau der Residenz vom Vormärz bis zum Ende des „Kaiserforums“. Wien: Verlag der Österreichischen Akademie der Wissenschaften (Veröffentlichungen der Kommission für Kunstgeschichte: 15).

18 Allgemeines Bürgerliches Gesetzbuch (1811). 


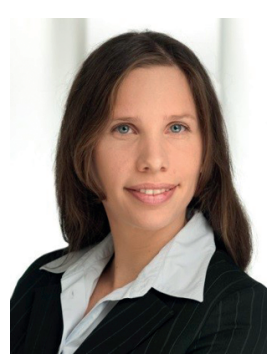

Nina Knieling

Österreichische Nationalbibliothek Josefsplatz 1

A-1015 Wien

Österreich

knieling@ponte.at

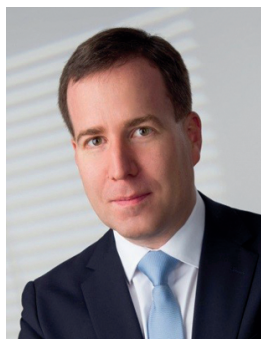

Thomas Huber-Frischeis

Österreichische Nationalbibliothek Josefsplatz 1

A-1015 Wien

Österreich

thomas.huber@onb.ac.at

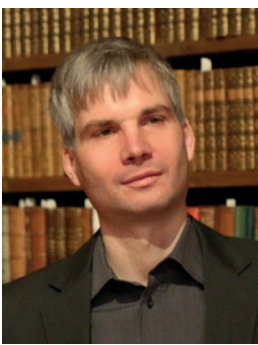

Rainer Valenta

Österreichische Nationalbibliothek Josefsplatz 1

A-1015 Wien

Österreich

rainer.valenta@onb.ac.at

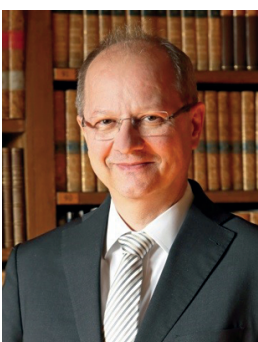

Dr. Hans Petschar

Österreichische Nationalbibliothek Bildarchiv und Grafiksammlung Josefsplatz 1

A-1015 Wien

Österreich

Hans.Petschar@onb.ac.at 